\title{
PWWP Domain
}

National Cancer Institute

\section{Source}

National Cancer Institute. PWWP Domain. NCI Thesaurus. Code C14044.

Around 70 amino acids and weakly conserved, the PWWP Domain is named after a conserved Pro-Trp-Trp-Pro motif often found as multiple copies in proteins that contain chromatin-association domains or in cell growth and differentiation transcription factors, such as WHSC1 (C-terminus) and ASH1/T rithorax Group proteins. Although its function is unknown, the amino acid composition suggests that it may be involved in protein-protein interactions. The domain $\mathrm{N}$-terminal half resembles a barrel-like five-stranded structure; the C-terminal half contains a five-helix bundle. The halves form a module that exhibits positive electrostatic potential. Alone, the domain binds DNA in vitro, probably through its basic surface. $(\mathrm{NCl})$ 